\title{
BMJ Open Living with Dementia in Aotearoa (LiDiA): a cross-sectional feasibility study protocol for a multiethnic dementia prevalence study in Aotearoa/ New Zealand
}

\author{
Adrian Martinez-Ruiz (D) , ${ }^{1,2}$ Susan Yates, ${ }^{1}$ Gary Cheung, ${ }^{1}$ Makarena Dudley, ${ }^{3}$ \\ Rita Krishnamurthi, ${ }^{4}$ Fuafiva Fa'alau, ${ }^{5}$ Mary Roberts, ${ }^{6}$ Seini Taufa, ${ }^{6}$ \\ Jacinta Fa'alili-Fidow, ${ }^{6}$ Claudia Rivera-Rodriguez, ${ }^{7}$ Staverton Kautoke, ${ }^{8}$ \\ Etuini Ma'u, ${ }^{1}$ Ngaire Kerse, ${ }^{5}$ Sarah Cullum (1) ${ }^{1}$
}

To cite: Martinez-Ruiz A, Yates S, Cheung G, et al. Living with Dementia in Aotearoa (LiDiA): a cross-sectional feasibility study protocol for a multiethnic dementia prevalence study in Aotearoa/ New Zealand. BMJ Open 2021;11:e046143. doi:10.1136/ bmjopen-2020-046143

- Prepublication history for this paper is available online. To view these files, please visit the journal online (http://dx.doi. org/10.1136/bmjopen-2020 046143).

Received 26 October 2020 Revised 16 March 2021 Accepted 20 April 2021
Check for updates

(C) Author(s) (or their employer(s)) 2021. Re-use permitted under CC BY-NC. No commercial re-use. See rights and permissions. Published by BMJ.

For numbered affiliations see end of article.

Correspondence to

Dr Sarah Cullum;

sarah.cullum@auckland.ac.nz

\section{ABSTRACT}

Introduction Aotearoa/New Zealand (NZ) is officially recognised as a bicultural country composed of Māori and non-Māori. Recent estimations have projected a threefold increase in dementia prevalence in NZ by 2050 , with the greatest increase in non-NZ-Europeans. The NZ government will need to develop policies and plan services to meet the demands of the rapid rise in dementia cases. However, to date, there are no national data on dementia prevalence and overseas data are used to estimate the NZ dementia statistics. The overall aim of the Living with Dementia in Aotearoa study was to prepare the groundwork for a large full-scale NZ dementia prevalence study.

Methods and analysis The study has two phases. In phase I, we will adapt and translate the 10/66 dementia assessment protocol to be administered in Māori, Samoan, Tongan and Fijian-Indian elders. The diagnostic accuracy of the adapted 10/66 protocol will be tested in older people from these ethnic backgrounds who were assessed for dementia at a local memory service. In phase II, we will address the feasibility issues of conducting a populationbased prevalence study by applying the adapted 10/66 protocol in South Auckland and will include NZ-European, Māori, Samoan, Tongan, Chinese and Fijian-Indian participants. The feasibility issues to be explored are as follows: (1) how do we sample to ensure we get accurate community representation? (2) how do we prepare a workforce to conduct the fieldwork and develop quality control? (3) how do we raise awareness of the study in the community to maximise recruitment? (4) how do we conduct door knocking to maximise recruitment? (5) how do we retain those we have recruited to remain in the study? (6) what is the acceptability of study recruitment and the 10/66 assessment process in different ethnic groups?

Ethics and dissemination The validity and feasibility studies were approved by the New Zealand Northern A Health and Disability Ethics Committee (numbers 17NTA234 and 18NTA176, respectively). The findings will be disseminated through peer-reviewed academic

\section{Strengths and limitations of this study}

- This is the first study conducted by bilingual and bicultural researchers to test a methodology aimed to determine the feasibility of conducting a multiethnic dementia prevalence study in Aotearoa/New Zealand (NZ).

- The study will use strict guidelines to adapt, translate and validate the 10/66 dementia assessment instrument in four of the largest ethnic groups living in Aotearoa/NZ.

- The study will use meshblock census data and oversampling to ensure inclusive representation of the non-European ethnic groups included in the study.

- Due to time and funding constraints, we will include only the major NZ ethnic groups in this feasibility study.

- We will use door knocking to ascertain our sample; other sampling methods such as using the electoral roll may be more effective but are unlikely to be accurate in the geographical area we have chosen.

journals, national and international conferences, and public events. Data will be available on reasonable request from the corresponding author.

\section{INTRODUCTION}

Dementia is a global health priority and its prevalence is increasing in many parts of the world due to their ageing populations. ${ }^{1}$ Governments across the world are developing policies and planning services to meet the healthcare and social needs of people with dementia. Aotearoa/New Zealand (NZ) needs to do the same, but to date, there are no prevalence data at a national level to inform the extent and impact of dementia on our population. Although it has been projected 
that over 60000 people are currently living with dementia in NZ (at a cost of $\$ 1700$ million per annum) and it will increase to 170000 people by $2050,{ }^{2}$ these statistics are extrapolated from other countries data.

Globally, the prevalence of dementia in people aged 60 years and over is reported to be between $5.6 \%$ and $7.6 \% .^{3}$ However, the prevalence of dementia and risk factor profiles may be different among different ethnic groups within the same population. For example, the prevalence of dementia in Aboriginal Australians is three times higher than their non-indigenous counterparts; and head injury has been identified as a risk factor that is significantly associated with dementia in Aboriginal Australians. ${ }^{5}$ Other studies have included multiethnic samples. ${ }^{6}$ For example, a study conducted in Singapore used the $10 / 66$ assessment protocol ${ }^{7}$ to calculate the prevalence of dementia among their population. ${ }^{6}$ It included a sample of 2565 subjects aged 60 years and over who speak Chinese, Malay, Tamil or other dialects (Hokkien, Cantonese and Teochew). The instrument was first translated and adapted into those languages that had not been translated before and subsequently applied. The results showed an overall dementia rate of $10 \%$ using the 10/66 diagnostic algorithm. ${ }^{6}$ Interestingly, they also found that the Indian population had a lower probability of having dementia compared with the Chinese-speaking population. ${ }^{6}$ These results demonstrate how the prevalence and aetiologies may vary in different populations. Therefore, careful assessment of each population is essential to establish both the prevalence of dementia and communityspecific risk factors related to it.

Aotearoa/NZ is officially recognised as a bicultural country which includes Māori and non-Māori people. Non-Māori people are composed of NZ-Europeans, Asian, Pacific People, Middle Eastern, Latin American and Africans. The Treaty of Waitangi is NZ's constitutional document that places the obligation on the $\mathrm{NZ}$ government to be responsive to the health needs of Māori, including those living with dementia and to ensure equitable health outcomes with non-Māori. ${ }^{8}$ According to the $2018 \mathrm{NZ}$ census, approximately $70 \%$ of the people in the total population self-identified as NZ-Europeans, $17 \%$ as Māori, $15 \%$ as Asians, $8 \%$ as Pacific people, $2 \%$ as Middle Eastern/Latin American/African and 1\% as others. ${ }^{9}$ However, the $2018 \mathrm{NZ}$ census also included those who identify with more than one ethnicity; thus, the proportion sum is higher than $100 \%$. Also, in urban areas of NZ, there are many diverse communities in which a large proportion of the people are not able to speak English as reported in the Counties Manakau Population Census $^{10}$ (table 1). This might be explained by recent NZ immigration policies in which many older people from these ethnic groups emigrated to $\mathrm{NZ}$ following their adult children in the last 20 years; therefore, many have had no need to learn English prior to immigration. Also, they often live in close-knit communities, speaking their mother tongue in everyday life, and hence usually there is no need to learn English after their arrival in NZ.
Table 1 Language indicator for the 2013 Counties Manakau Population Census in people aged 65 years and over

\begin{tabular}{lll}
\hline $\begin{array}{l}\text { Ethnic } \\
\text { group }\end{array}$ & $\begin{array}{l}\text { People who are } \\
\text { not able to speak } \\
\text { English (\%) }\end{array}$ & $\begin{array}{l}\text { People who are able } \\
\text { to speak their own } \\
\text { language (\%) }\end{array}$ \\
\hline Chinese & 64 & $90^{\star}$ \\
Indian & 37 & $56 \dagger-85 \ddagger$ \\
Samoan & 35 & 97 \\
Tongan & 44 & 92 \\
\hline
\end{tabular}

Totals do not add to a hundred per cent as people might be included in one or more categories, and not all categories included in the report were described in this table.

${ }^{*}$ Corresponds to the total of people able to speak a Sinitic language.

†Corresponds to people who are able to speak Hindi.

$\ddagger$ Corresponds to people who are able to speak Indian languages other than Hindi.

The prevalence of dementia is likely to be different among the major ethnic groups in NZ. For example, the largest ethnic minorities (Māori, Asian and Pacific populations) are increasing at a faster rate than NZ-Europeans, ${ }^{2}$ and we will expect a higher increase in dementia prevalence in these populations. There is also some evidence to suggest Māori and Pacific people are diagnosed with dementia at a younger age than NZ-Europeans, ${ }^{11}$ possibly due to their higher rates of cardiovascular risk factors. ${ }^{12} 13$ A previous study found that Asian people living in NZ are more likely to have their dementia undiagnosed, and their true prevalence of dementia might be higher than what is reported in official reports. ${ }^{14}$

Accurate estimates of dementia prevalence and associated risk factors in NZ are critical to measure the full impact of dementia, raise public awareness, reduce stigma and inform policy development regarding the implementation of evidence-based prevention, treatment and support services for people with dementia and their families. ${ }^{15}$ Culturally appropriate and responsive services for dementia can only be developed if the true extent of the burden of dementia is known. There has never been a population-based dementia prevalence study in NZ, so we propose to test the methods required to conduct a fully representative multiethnic national prevalence study of dementia. The aim of this paper was to describe the study protocol of a validity study and a feasibility study that will prepare the groundwork for a future fully powered dementia prevalence study in NZ (figure 1).

There is an inherent educational and cultural bias in many cognitive tests that were developed for European cultures. Thus, commonly used English dementia instruments are not appropriate to apply in those communities, and there is a need to use fully adapted and validated instruments that can produce accurate and comparable ethnic-specific rates for a NZ dementia prevalence study. Due to the multicultural setting in NZ, we elected to use the 10/66 dementia assessment protocol, which was 


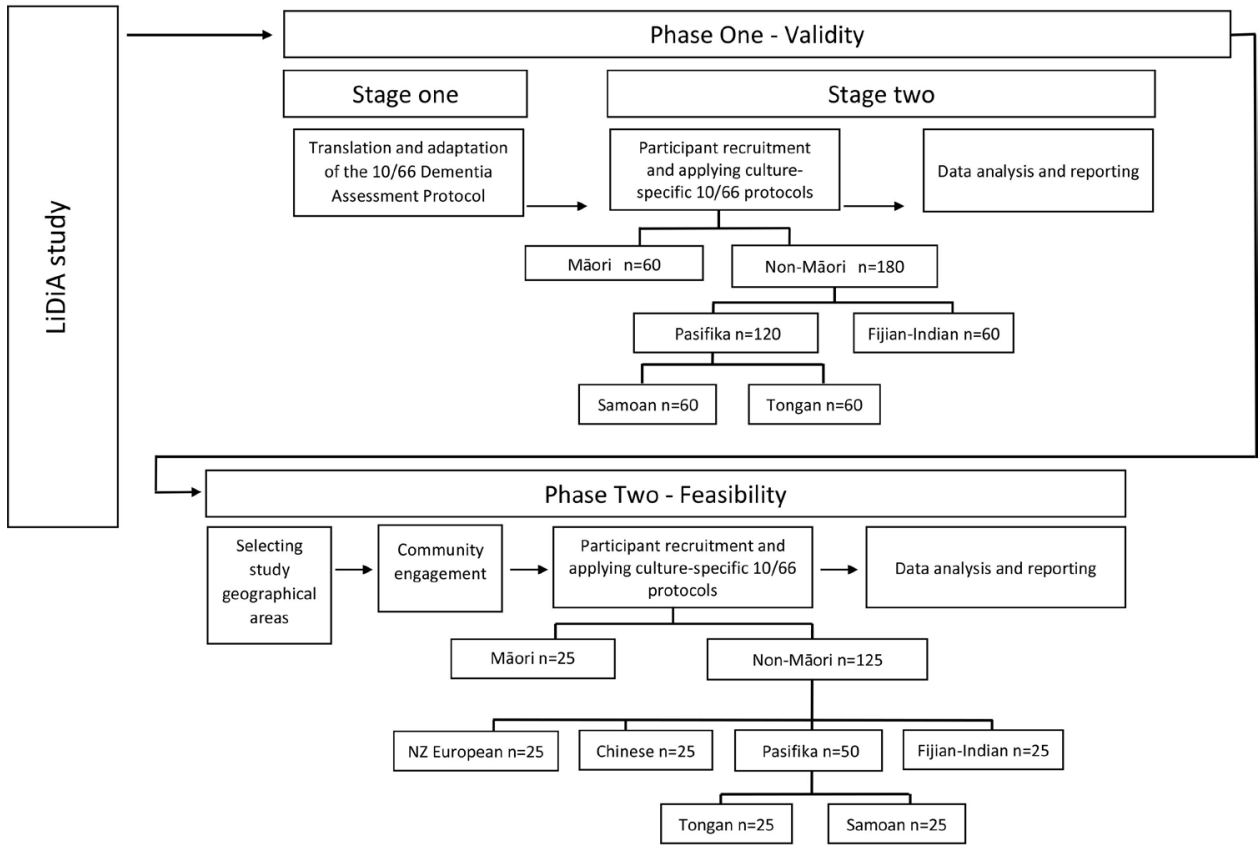

Figure 1 LiDiA feasibility study design. LiDiA, Living with Dementia in Aotearoa.

developed to be culturally and linguistically fair and can be administered by trained lay interviewers. ${ }^{16}$ The $10 / 66$ protocol has been demonstrated to have a sensitivity of up to $94 \%$ and a specificity of up to $97 \%$ in diagnosing dementia. ${ }^{17}$ It has been previously translated and validated in many languages, including Spanish, Portuguese, Mandarin and Cantonese. ${ }^{17}$ However, it has never been used in Māori or Pacific populations.

The objectives of the validity study were (1) to translate and adapt the 10/66 protocol for use in research with Māori, Samoan, Tongan and Fijian-Indian (people of Indian ethnicity born in Fiji Islands) populations; and (2) to test the diagnostic accuracy of these adapted versions in the respective ethnic groups.

The objective of the feasibility study was to test the logistics and feasibility of using the culturally adapted versions of the 10/66 protocol as a research tool with NZ-European, Māori, Chinese, Samoan, Tongan and Fijian-Indian people living in the community. The results of the feasibility study will inform a future fully powered dementia prevalence study in NZ.

The largest minority ethnic groups (Samoan, Tongan, Fijian-Indian and Chinese) will be included in the study based on the availability of bilingual bicultural researchers and interviewers from the same ethnic groups.

\section{METHODS AND ANALYSIS}

\section{Phase I: validity study}

Stage 1: translation and adaptation of the 10/66 dementia assessment protocol

The 10/66 dementia assessment protocol takes approximately 90 min to administer, the main sections of which are described in table 2.
We will adopt a translation procedure based on the WHO translation guidelines. ${ }^{18}$ The procedure entails a four-stage process:

1. Forward translation: the English version of the 10/66 will be translated into Te Reo Māori (the Māori language), Samoan, Tongan and Fijian Hindi by professional translators, assisted by a clinician.

2. Expert advisory panels for the selected ethnicities will review the first draft of the translation and offer advice and possible solutions for acceptability, conceptual validity and tolerability of the translated instruments.

3. A bilingual dementia specialist will quality check and back translate the adapted/translated version.

4. Pretesting in individuals with and without dementia and their families will be performed to assess how well the questionnaire will be received, and their feedback will be used to refine the final version.

We acknowledge the importance of building a research team that represents the ethnic backgrounds of the population groups we are seeking to engage for the study. Māori, Samoan, Tongan and Fijian Hindi researchers will assist in the recruitment of expert translators and members for each expert advisory panel and facilitate the meetings.

\section{Stage 2: diagnostic accuracy}

We will recruit Māori, Samoan, Tongan and Fijian-Indian participants from the Counties Manukau District Health Board (CMDHB) memory service based at Middlemore Hospital in South Auckland, NZ. People who attend the memory service are referred either from primary care or secondary care services. The clinical criteria to access this service is that a person and/or their family living in the 


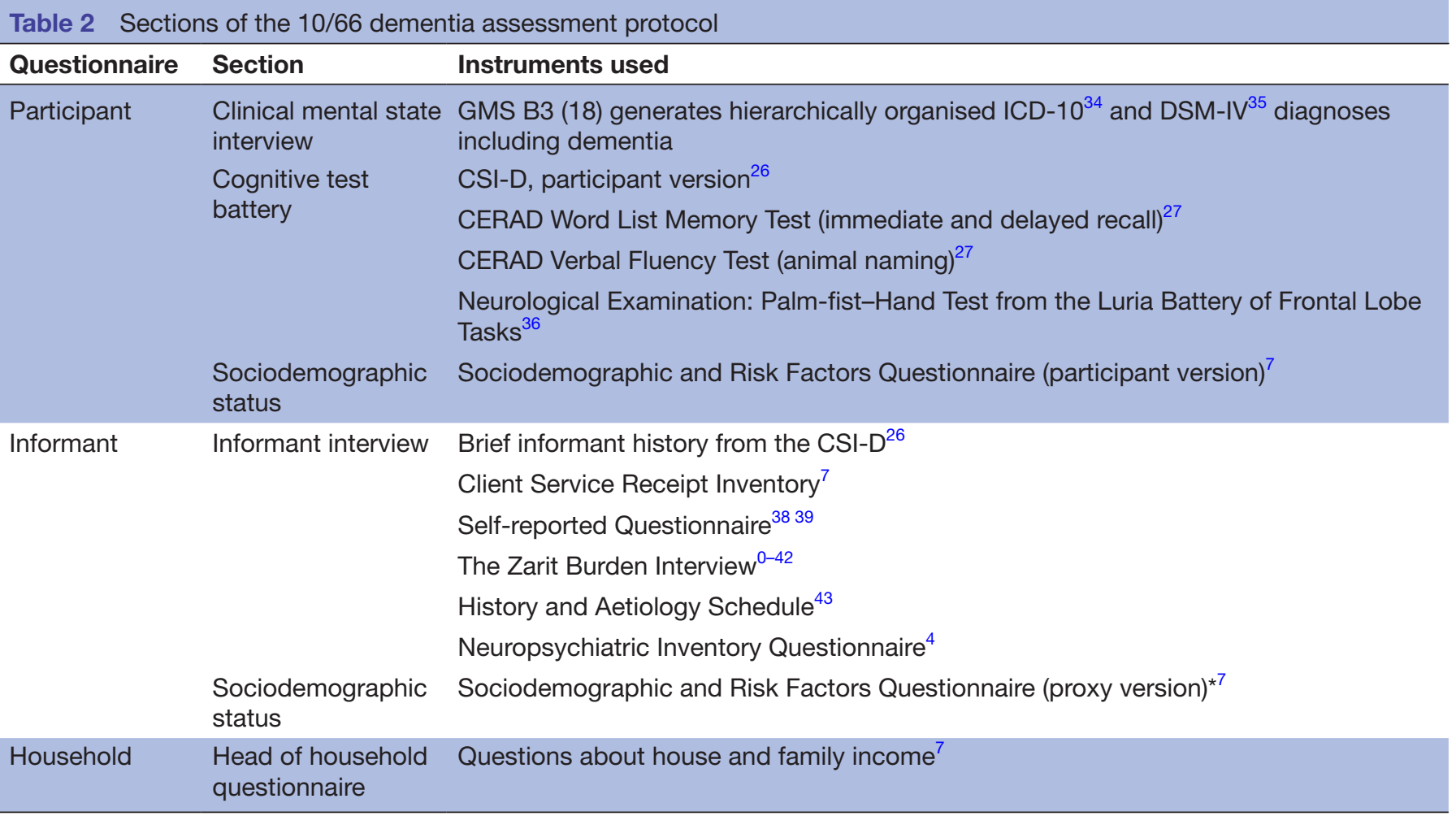

*Proxy version will be used if the main participant is unable to complete the participant version of the Sociodemographic and Risk Factors Questionnaire.

CERAD, Consortium to Establish a Registry for Alzheimer's Disease; CSI-D, Community Screening Interview for Dementia; DSM IV, Diagnostic and Statistical Manual of Mental Disorders, Fourth Edition; GMS, Geriatric Mental State; ICD, International Classification of Diseases.

community have a primary concern of subjective and/or objective cognitive decline, irrespective of age.

To assess diagnostic test accuracy, we will compare the results of the 10/66 dementia assessment protocol with the clinical diagnoses. The clinical diagnoses will be made by a multidisciplinary team of dementia specialists at the memory service, guided by standard clinical criteria, including the National Institute on Aging and Alzheimer's Association (NIA-AA) criteria for Alzheimer's disease dementia, ${ }^{19}$ the National Institute of Neurological Disorders and Stroke and the Association Internationale pour la Recherche et l'Enseignement en Neurosciences (NINCDS-AIREN) criteria for vascular dementia, ${ }^{20}$ criteria for Lewy Body dementia ${ }^{21}$ and the clinical criteria for frontotemporal dementias. ${ }^{22}$ Dementia severity is guided by the Criteria for Dementia Severity $(\mathrm{CDR})^{23}$; a CDR of 0.5 indicates mild cognitive impairment; 1 is mild severity; 2 and 3 are moderate and severe severity. ${ }^{23}$

\section{Cases and controls}

Eligible participants in the validity study will be 65 years or older and self-identify as Māori, Samoan, Tongan or FijianIndian. Participants must have had a full dementia assessment at the CMDHB memory service. The assessment or review must have taken place in the previous 6 months to avoid the potential progression of normal controls converting to dementia. By using convenience sampling, we will attempt to recruit a total of 30 participants with a dementia diagnosis in each of the four ethnic groups, and 30 age-matched and sex-matched controls (who had a full specialist assessment and were found not to have dementia). Participants will be excluded if they suffer from any physical or sensory impairment that compromises their ability to participate in the interview.

\section{Informants}

All participants will have a family member or main caregiver (informant) who will complete the 'informant' section of the 10/66 protocol (table 2). An informant is defined as a person who knows the main participant well.

\section{Blinding}

The selection, recruitment and clinical confirmation of case/control status will be carried out independent of the 10/66 interviewing process. Interviewers will be blind to the case or control group to which the participant belongs, although blinding may be difficult to maintain in cases of more severe dementia.

\section{Interview training and assessment process}

Lay interviewers will be recruited via electronic resources (university website, email and blogs) or through contacts from people/students/health professionals known to the study's investigators. Interviewers must be bicultural and bilingual, identify with at least one of Māori, FijianIndian, Tongan or Samoan ethnicity, and be able to speak 
English and the ethnic group language fluently. We will select between two and six interviewers per ethnic group. The interviewers will be trained by a team of researchers familiar with the 10/66 assessment protocol. The lead coinvestigator for each ethnic group will ensure that specific cultural guidelines are included in the training process. The training will consist of four sessions of approximately 3 hours each. In the first session, the 'participant' questionnaires and cognitive tests will be reviewed. In the second session, the informant and 'head of household' questionnaires, consent forms and participant information sheets will be reviewed. During the third session, interviewers will undergo training on specific protocols to handle unexpected situations. For example, if mental health problems are detected during the interview (eg, suicidal ideation), the principal investigator will be immediately contacted by the interviewers, and after assessing the situation and with their prior consent, the participant's/informant's general practitioner or the appropriate mental health service will be contacted to inform them about the issue. In the fourth session, answers will be provided to clarify any questions that the interviewers may have had during the training process, in particular, cultural and language issues.

\section{Interviews}

After obtaining written consent, interviewers will conduct the assessment in a manner that is culturally appropriate and follows the respective cultural protocols. For example, in Māori whānau (families), at the beginning of any hui (social gathering) and following the karakia (prayer), a round of mihimihi (introductions and speeches) usually occurs. During this time, people may share information about where they come from and significant aspects of their whakapapa (genealogy). The interview is usually finished with another karakia. Similarly, for Pacific families, the importance of taking time to build trust and rapport requires the incorporation of Samoan and Tongan customs-'fa'aSamoa' / anga fakaTonga' and an inherent understanding of Pacific values such as 'Tausi le va' /'Tauhi le va' (nurturing relationships) and 'fa'aaloalo'/'faka'apa'apa' (respect), which have specific connotations for interactions and engagement with the elderly. On completion of the assessment session, a koha (gift) of a NZ $\$ 100$ voucher will be offered to the person and their family as a gesture of appreciation for their participation. The Geriatric Mental State $(\mathrm{GMS})^{24}$ will assess if the participant was not interviewed in their mother language or if the participant was using an unclear dialect or accent. We presume that some of the participants will be to some extent bilingual; however, this will depend on the characteristics of their life history and other sociocultural factors. Since the interviewers will be bilingual, the participants will be able to decide in which language they prefer the interview to take place.

For participants who are unable to give fully informed consent, we will follow the process recommended by the NZ's Code of Health and Disability Services Consumers'
Rights. ${ }^{25}$ We will approach the family to discuss whether they believe their family member would want to participate. If the caregiver agrees, we will seek written confirmation that they have been consulted, and are comfortable with the researcher making the decision as to whether their relative should participate in the study, and that they believe this would be consistent with their relative's wishes. If at any time the participants indicate they do not wish to participate, the interview will be terminated.

\section{Analysis phase I}

The predictive analytical software SPSS V.25 will be used for data analysis. Descriptive frequency distributions and mean values will be used to describe the demographic summary of each ethnic group. Dementia diagnosis will be made using the 10/66 dementia diagnostic algorithm, which has been described elsewhere, ${ }^{17}$ but in brief, the algorithm uses the scores obtained from (1) the Community Screening Instrument, ${ }^{26}$ (2) the Verbal Fluency Test, ${ }^{27}$ (3) the Consortium to Establish a Registry for Alzheimer's Disease word list memory test, ${ }^{27}$ and (4) the scores/diagnoses obtained from the GMS interview. ${ }^{24}$ The algorithm is processed in two sequential stages: in stage 1, the total scores for each component are calculated, and in stage 2 , the final diagnoses are arranged by a hierarchically structured imposed algorithm. ${ }^{717}$ In order to obtain the positive predictive value, negative predictive value, specificity and sensitivity, the resulting dichotomous variable derived from the algorithm will be assessed against the gold standard clinical diagnoses of 'dementia case' or 'no dementia case'. To compare the subcomponents of the 10/66 protocol between cases and controls, descriptive analyses of categorical variables will be assessed using $\chi^{2}$ tests, and those of normally distributed continuous variables will be assessed with Student's t-test or one-way analysis of variance. Non-parametric variables will be assessed with Mann-Whitney U test or Kruskal-Wallis.

\section{Phase II: feasibility study}

Phase II is designed to answer the feasibility questions that arise when we attempt to use the culturally adapted 10/66 dementia assessment tools in community-dwelling participants living in selected geographical areas. South Auckland was selected for this purpose due to its ethnic diversity. The feasibility study will include the following ethnic groups: NZ-European, Māori, Fijian-Indian, Chinese, Tongan and Samoan.

The six main feasibility questions to be answered are

1. How do we sample to ensure we get adequate community representation from the included ethnic groups?

2. How do we prepare a workforce to conduct the fieldwork and develop quality control?

3. How do we raise the awareness of the study in the community to maximise recruitment?

4. How do we conduct door knocking to maximise recruitment?

5. How do we retain those we have recruited to remain in the study? 
6. What is the acceptability of study recruitment and the $10 / 66$ assessment process in different ethnic groups?

The design of the feasibility study will replicate the design of the full dementia prevalence study. Potential participants will be identified and recruited using door knocking in the selected areas that will allow oversampling of non-European ethnic groups. We will include those aged 65 years or over living in private residences in the selected areas and their caregivers/coresidents. The exclusion criteria are participants unable to identify a friend or family member to complete the informant schedule, and people living in long-term care facilities and retirement villages (since their overall dementia prevalence, sociodemographic and general health status may differ from those in the community, and thus it might introduce results bias in our relatively small community sample). The same procedures regarding the interview and interviewer training process will be used for the feasibility study. All stored data will be deidentified and coded with a unique participant identifier.

\section{Feasibility issues}

\section{Sampling to ensure adequate community representation from the} included ethnic groups

This phase is a cross-sectional survey of selected ethnicities identified by a standard procedure for a populationbased sample. It includes a meshblock sampling frame and door-to-door knocking to recruit a representative sample in the selected areas. ${ }^{28}$ Meshblocks are defined as the smallest geographical unit for which Statistics NZ has demographic information (approximately 100 people). Using NZ Census demographic information and the expected rates of dementia by age, we will calculate the probability of finding dementia cases in the community in adults aged 65 years or older in the CMDHB region of South Auckland. We will then test our sampling methods by selecting meshblocks with the highest proportions of people aged $65+$ years for each ethnicity. We aimed to recruit 25 participants and informants from each of the groups, enabling us to test the study procedures and materials in all six ethnic communities.

\section{Preparing a workforce to conduct the fieldwork and developing quality control}

The interviewers will be fully trained as described in phase I of the study. Quality control processes will be conducted before and throughout the study interviews. Interviewers will practice interviews with volunteers aged 65 years or older, without significant medical or psychiatric comorbidity. The volunteer interviews will be carried out during the training process and constructive feedback regarding their approach and the conduct of the interview will be provided.

After the training sessions, the first two study interviews will be carried out under the supervision of one of the trainers, and specific feedback regarding the interview process will be provided at the end of the interview. This will ensure that the 10/66 assessment protocol is correctly administered across all the different ethnic groups and will clarify any questions that may arise during the interview process.

Raising awareness of the study in the community: participant and public involvement

Three to four months before starting the sample recruitment, we will engage with the selected communities to codesign a study launch strategy in the communities we hope to reach using traditional media, social media and ethnic-specific community activities cofacilitated with community leaders. We will also send information about the study by post to all potential households in the chosen locations. Subsequently, we will ask study participants to feedback if/how they knew about the study beforehand, informing our launch strategy for the full study. Once the community engagement activities are completed, we will start the recruitment of potential participants.

\section{Maximising recruitment by door knocking}

The feasibility study will use a door-to-door recruitment approach. Door knocking will be conducted at least once a week for 12 months in the selected areas or until a response has been recorded for each household. The door-knocking team will be composed of bilingual and bicultural interviewers (at least one per ethnic group). The initial questionnaire will be conducted on the doorstep and will last approximately $10 \mathrm{~min}$. If inclusion criteria are met, the study will be briefly described to the potential participant and, if agreed, we will ask them for contact details to send further information about the study. The door knockers will return to households up to four times to maximise response rates before registering a house as 'not answered'. Regardless of whether or not they agree to participate in the study, all participants who answer our initial questions will receive a koha/gift (a key ring) as a gesture of appreciation for their time.

Information about the study will be sent by mail to the potential participants who have agreed to be contacted. They will be given the contact phone details of the lead coinvestigator for their ethnic group if they wish to discuss the study further. Approximately 1 week after sending these documents, a phone call will be made, with the aim of answering any questions that the potential participant might have and to make an appointment to carry out the interview. The call will be made by a bilingual bicultural researcher which will reduce any potentially coercive power differential, facilitating cultural safety. Interviews will be carried out either at the participant's home, University of Auckland facilities or other suitable location of their choice.

\section{Retaining those we have recruited to the study}

Once the participant/informant agrees to participate in the study, he/she will be contacted to schedule an appointment. Appointments will be arranged by the ethnic-specific lead coinvestigator or by someone trained and designated by them (eg, one of the bilingual 
interviewers). We will contact the participants either by phone call or face-to-face, depending on which is most culturally appropriate. The contact will be made in the participant's native language or in English, depending on the participants' preferences. The interviewer will fully explain the study to all potential participants (including the head of household and family member participants) and will discuss any concerns. If they agree to participate, we will seek informed consent from the participant, the informant and the household informant. Regular staff meetings will be held with interviewers to obtain feedback that will inform the adaptation of study protocols. We will also test our fieldwork protocol that includes verification of ineligible/refusal cases, contact with families to ask and measure that correct protocol was followed, quality control of data and observation of interviews.

\section{Acceptability of study recruitment and assessment in different ethnic groups}

We will ask the participants and their families for feedback about the interview and the specific cultural approach. We will assess the consent procedures (eg, total time used to fill the consent form and answer questions about the consent form, and difficulties around signing/ reading the consent form); questionnaire administration (total time to finish the interview, appropriateness of the questions and participants' general opinions about the questionnaire); and koha (gift) management (best way to offer koha to participants and participants' opinions about the koha). Finally, we will ask interviewers to feed back problems that they encounter and will use this information to refine our recruitment, training procedures and fieldwork monitoring in the full study, and to help decide whether to outsource to a survey firm in the full study.

\section{Quantitative data collection and reporting}

Recruitment will be assessed by the number of people screened in the selected meshblocks. We will register the total number of door-knocked houses, number of door-knocked houses answered and declined, numbers of people over the age of 65 years that agreed to be contacted, the houses that agreed to be contacted but subsequently declined (and, if possible, the reasons for declining) as well as final interview response rate. For those who agreed to be contacted, we will measure the retention rate, decline rate, proportion of baseline participants that agree to be interviewed and the completeness of collected data.

Overall, we will measure the proportion of potential participants (and informants) who were approached, consented and completed the research protocol and adapted 10/66 interview schedule as a quantitative measure of acceptability.

These measures will enable us to test the effectiveness of the sampling procedure and we will compare our results to NZ Census data to assess whether we reached a representative sample of the South Auckland population.

\begin{tabular}{|c|c|c|}
\hline $\begin{array}{l}\text { Study } \\
\text { phases }\end{array}$ & Tasks & Months \\
\hline \multirow[t]{3}{*}{$\begin{array}{l}\text { Validity } \\
\text { study }\end{array}$} & $\begin{array}{l}\text { Adaptation of } 10 / 66 \text { dementia } \\
\text { protocols for specific cultures }\end{array}$ & $1-3$ \\
\hline & $\begin{array}{l}\text { Participant recruitment and interview } \\
\text { using culture-specific 10/66 protocols }\end{array}$ & $4-7$ \\
\hline & Data analysis and report writing & $8-12$ \\
\hline \multirow{3}{*}{$\begin{array}{l}\text { Feasibility } \\
\text { study }\end{array}$} & Door knocking & $8-12$ \\
\hline & $\begin{array}{l}\text { Participant recruitment and interview } \\
\text { using culture-specific 10/66 protocols }\end{array}$ & $13-20$ \\
\hline & Data analysis and report writing & $20-24$ \\
\hline
\end{tabular}

The numbers of people with dementia identified in each ethnic group will inform the sample size and the weighted stratification method, which is usually used to estimate prevalence in under-represented groups in the population in the fully powered dementia prevalence study.

The data collection and analysis timeline are described in table 3 .

\section{Patient and public involvement}

Before starting the study, we will engage with the selected communities to conduct educational sessions and also conduct qualitative research to identify the main concerns about dementia in each ethnic group. Other sessions have already been conducted through local non-governmental organisations serving older people in different ethnic groups living in NZ, plus cross-cultural interest groups with Asian health professionals regarding the best ways to conduct dementia research in their communities. ${ }^{29} \mathrm{We}$ will also invite members from the different communities to a study launch.

\section{DISCUSSION}

There has never been a population-based dementia prevalence study in NZ. Thus, we do not have a clear idea of the true extent and impact of dementia both overall and, in particular, in Māori and Pacific people who may be at greater risk yet remain undiagnosed. A dementia prevalence study that represents all major ethnic groups in NZ is needed to (1) measure the true extent of dementia in $\mathrm{NZ}$, (2) examine the risk factor profiles in each ethnic group, (3) measure the care arrangements and caregiver burden in families living with dementia, and (4) determine the economic impact of dementia on families and on society. The major impact of this study is the creation of new knowledge about the community prevalence of dementia in NZ, both overall and for all major ethnic groups, which is essential to inform culturally appropriate strategies to reduce the impact of dementia.

There are some limitations that need to be acknowledged: 
1. The sampling methodology was based on convenience sampling. Although sample sizes between 24 and 50 have been recommended for pilot studies ${ }^{30-32}$ and convenience sampling may provide accurate correlations and rich qualitative information, it will not offer generalisable results to the overall NZ population. However, this study will lay the foundations for a future national prevalence study representing all the ethnic groups included in our research.

2. Not all ethnic minorities in NZ will be included in this phase of the study; other ethnic groups will need to be included in future studies, for example, people from other Pacific Islands, Middle Easterners, Latin Americans and Africans.

3. Another limitation is that we will only include people aged 65 years or over. Future studies, including people with younger-onset dementia, particularly from the ethnic groups that have been shown to be at a higher risk of developing dementia at a younger age (such as Maori and Pacific People), will be needed to clarify this issue.

4. The feasibility phase will only include people recruited from the community. Consequently, people living in long-term care facilities and retirement villages will be excluded from our study. However, we intend to conduct a future study using the long-term care facility version of the International Residential Assessment Instrument (interRAI). ${ }^{33}$ interRAI routinely collects information on dementia diagnosis and is mandated by the Ministry of Health to be completed with every long-term care facility residents every 6 months. We also have planned to conduct a dementia prevalence study in long-term care facilities using the 10/66 instruments (reference standard) and compare the results against interRAI data to assess their utility for ongoing dementia surveillance.

5. Finally, our study will be carried out in a multiethnic urban area. Nevertheless, in less ethnically diverse regions, it may be more challenging to recruit bilingual interviewers for ethnic minorities other than Maori. It would mean looking for alternatives to recruit interviewers for these populations-for example, mobilising bilingual interviewers from one location to another-which will increase the study costs but will present more accurate results. Additionally, in rural areas, it is likely that different engaging strategies will have to be sought. For example, disseminating the study in a rural population might require other engaging methods (such as face-to-face) compared with the methods used in urban areas. Also, due to cultural factors, the participation and declining rates might be different from less to more ethnically diverse areas and from rural to urban areas. A specific engaging method will have to be tested for these areas.

The findings from the full prevalence study will provide robust evidence about the numbers of people affected, the possible risk factors, caregiver burden and the financial impact on families. These NZ-specific data can be used by the NZ Ministry of Health to develop culturally informed policies to raise public awareness about dementia and dementia prevention and to plan services that support families living with dementia in all NZ communities. The study will also demonstrate the benefits of recruiting a qualified, skilled research team that is representative of the families participating in the study. Taken together, this study will determine the essential elements required for conducting dementia research in a multicultural context in NZ.

\section{ETHICS AND DISSEMINATION}

The validity study was approved by the Northern A Health and Disability Ethics Committee (number 17NTA234), and the feasibility study was approved by the Northern A Health and Disability Ethics Committee (number 18NTA176). The findings will be disseminated through peer-reviewed academic journals, national and international conferences, and public events.

\section{Author affiliations}

${ }^{1}$ Department of Psychological Medicine, The University of Auckland, Auckland, New Zealand

${ }^{2}$ Departamento de Epidemiología Demográfica y Determinantes Sociales, Instituto Nacional de Geriatria, Ciudad de Mexico, Mexico

${ }^{3}$ School of Psychology, The University of Auckland, Auckland, New Zealand

${ }^{4}$ National Institute for Stroke and Applied Neurosciences, Auckland University of Technology, Auckland, New Zealand

${ }^{5}$ School of Population Health, The University of Auckland, Auckland, New Zealand

${ }^{6}$ Research Department, Moana Research, Auckland, New Zealand

${ }^{7}$ Department of Statistics, The University of Auckland, Auckland, New Zealand

${ }^{8}$ Department of Mental Health Services for Older People, Counties Manukau District Health Board, Auckland, New Zealand

Contributors AM-R, SC and GC drafted the manuscript and all coauthors critically revised the manuscript. SC, RK, GC, MD and NK contributed to the study design. $\mathrm{SC}, \mathrm{CR}-\mathrm{R}, \mathrm{NK}, \mathrm{SY}$ and GC contributed to the study analysis design. AM-R, SC, GC, MD, RK, FF, MR, ST, JF-F, CR-R, SK, EM, NK and SC contributed to the study development.

Funding This work was supported by the Health Research Council of New Zealand (grant number 18/590), the Brain Research New Zealand Centre of Research Excellence (feasibility study grant number 3715238) and a Counties Manukau District Health Board Tupu fund (grant number 498).

Competing interests None declared.

Patient and public involvement Patients and/or the public were involved in the design, conduct, reporting or dissemination plans of this research. Refer to the Methods and analysis section for further details.

Patient consent for publication Not required.

Provenance and peer review Not commissioned; externally peer reviewed.

Open access This is an open access article distributed in accordance with the Creative Commons Attribution Non Commercial (CC BY-NC 4.0) license, which permits others to distribute, remix, adapt, build upon this work non-commercially, and license their derivative works on different terms, provided the original work is properly cited, appropriate credit is given, any changes made indicated, and the use is non-commercial. See: http://creativecommons.org/licenses/by-nc/4.0/.

ORCID iDs

Adrian Martinez-Ruiz http://orcid.org/0000-0003-4974-6674

Sarah Cullum http://orcid.org/0000-0003-0785-9101 


\section{REFERENCES}

1 World Health Organisation. Dementia: a public health priority. Geneva: World Health Organization, 2012. https://apps.who.int/iris/ handle/10665/75263

2 Deloitte Report for Alzheimer's New Zealand. Updated dementia economic impact report 2016. New Zealand, 2017. Available: https://www.alzheimers.org.nz/getmedia/79f7fd09-93fe-43b0-a837771027bb23c0/Economic-Impacts-of-Dementia-2017.pdf/

3 Prince M, Wimo A, Guerchet M, et al. World Alzheimer report 2015: the global impact of dementia, an analysis of prevalence, incidence, costs and trends. London UK: Alzheimer's Disease International, 2015.

4 Li SQ, Guthridge SL, Eswara Aratchige P, et al. Dementia prevalence and incidence among the Indigenous and nonIndigenous populations of the Northern Territory. Med J Aust 2014;200:465-9.

5 Lo Giudice D, Smith K, Fenner S, et al. Incidence and predictors of cognitive impairment and dementia in Aboriginal Australians: A follow-up study of 5 years. Alzheimers Dement 2016;12:252-61.

6 Subramaniam M, Chong SA, Vaingankar JA, et al. Prevalence of dementia in people aged 60 years and above: results from the wise study. J Alzheimers Dis 2015;45:1127-38.

7 Prince M, Ferri CP, Acosta D, et al. The protocols for the 10/66 dementia research group population-based research programme. BMC Public Health 2007;7:165.

8 Dyall L. Dementia: continuation of health and ethnic inequalities in New Zealand. N Z Med J 2014;127:68-80.

9 Stats New Zealand Tatauranga Aotearoa. Ethnicity, 2018. Available: https://www.stats.govt.nz/topics/ethnicity

10 Winnard D, Lee M, Macleod G. Demographic profile: 2013 census, population of counties Manukau. Counties Manakau Health, 2013.

11 Cullum S, Mullin K, Zeng I, et al. Do community-dwelling Māori and Pacific peoples present with dementia at a younger age and at a later stage compared with NZ Europeans? Int J Geriatr Psychiatry 2018;33:1098-104.

12 Joshy G, Simmons D. Epidemiology of diabetes in New Zealand: revisit to a changing landscape. N Z Med J 2006;119:U1999.

13 Simmons D, Harry T, Gatland B. Prevalence of known diabetes in different ethnic groups in inner urban South Auckland. N Z Med J 1999;112:316-9.

14 Martinez-Ruiz A, Huang Y, Gee S, et al. Individual risk factors for possible undetected dementia amongst community-dwelling older people in New Zealand. Dementia 2020;19:750-65.

15 Livingston G, Sommerlad A, Orgeta V, et al. Dementia prevention, intervention, and care. Lancet 2017;390:2673-734.

16 Prince MJ. The 10/66 dementia research group - 10 years on. Indian J Psychiatry 2009;51:S8-15.

17 Prince M, Acosta D, Chiu H, et al. Dementia diagnosis in developing countries: a cross-cultural validation study. Lancet 2003;361:909-17.

18 World Health Organization. Process of translation and adaptation of instruments, 2020. Available: https://www.who.int/substance_abuse/ research_tools/translation/en/

19 Jack CR, Bennett DA, Blennow K, et al. NIA-AA research framework: toward a biological definition of Alzheimer's disease. Alzheimers Dement 2018;14:535-62.

20 Román GC, Tatemichi TK, Erkinjuntti T, et al. Vascular dementia: diagnostic criteria for research studies. Report of the NINDS-AIREN International workshop. Neurology 1993;43:250-60.

21 McKeith IG, Boeve BF, Dickson DW, et al. Diagnosis and management of dementia with Lewy bodies: fourth consensus report of the DLB Consortium. Neurology 2017;89:88-100.

22 Neary D, Snowden JS, Gustafson L, et al. Frontotemporal lobar degeneration: a consensus on clinical diagnostic criteria. Neurology 1998;51:1546-54.
23 Morris JC. Clinical dementia rating: a reliable and valid diagnostic and staging measure for dementia of the Alzheimer type. Int Psychogeriatr 1997;9:173-6.

24 Copeland JR, Dewey ME, Griffiths-Jones HM. A computerized psychiatric diagnostic system and case nomenclature for elderly subjects: GMS and AGECAT. Psychol Med 1986;16:89-99.

25 Code of health and disability services consumers' rights New Zealand: health \& disability commissioner/Te Toihau Hauora, Hauatanga, 1994. Available: https://www.hdc.org.nz/your-rights/ about-the-code/code-of-health-and-disability-services-consumersrights

26 Hall KS, Gao S, Emsley CL, et al. Community screening interview for dementia (CSI D); performance in five disparate study sites. Int J Geriatr Psychiatry 2000;15:521-31.

27 Morris JC, Heyman A, Mohs RC, et al. The consortium to establish a registry for alzheimer's disease (CERAD). Part I. Clinical and neuropsychological assessment of Alzheimer's disease. Neurology 1989;39:1159-65.

28 Rothman KJ, Greenland S, Lash TL. Modern epidemiology. 3rd edn. Philadelphia, USA: Lippincott Williams \& Wilkins, 2008.

29 Cheung G, Appleton K, Boyd M, et al. Perspectives of dementia from Asian communities living in New Zealand: a focus group of Asian health care professionals. Int J Geriatr Psychiatry 2019;34:1758-64.

$30 \mathrm{Sim}$ J, Lewis M. The size of a pilot study for a clinical trial should be calculated in relation to considerations of precision and efficiency. J Clin Epidemiol 2012;65:301-8.

31 Julious SA. Sample size of 12 per group rule of thumb for a pilot study. Pharm Stat 2005;4:287-91.

32 Browne $\mathrm{RH}$. On the use of a pilot sample for sample size determination. Stat Med 1995;14:1933-40.

33 Hirdes JP, Ljunggren G, Morris JN, et al. Reliability of the interRA suite of assessment instruments: a 12-country study of an integrated health information system. BMC Health Serv Res 2008;8:277.

34 World Health Organisation. The ICD-10 classification of mental and behavioural disorders: diagnostic criteria for research. Geneva: World Health Organization, 1993.

35 American Psychiatric Association. Diagnostic and statistical manual of mental disorders: DSM-IV. 4th edn. Washington, DC, 1994.

36 Luria AR, Haigh B. The working brain : an introduction to neuropsychology [London]. Harmondsworth: Allen Lane; Penguin, 1978.

37 Chisholm D, Knapp MR, Knudsen HC. Client socio-demographic and service receipt Inventory-European version: development of an instrument for international research. epsilon study 5 European psychiatric services: inputs linked to outcome domains and needs. Br J Psychiatry Suppl 2000;39:s28-33.

38 Martin AJ. Assessing the multidimensionality of the 12-item general health questionnaire. Psychol Rep 1999;84:927-35.

39 Mari JJ, Williams P. A comparison of the validity of two psychiatric screening questionnaires (GHQ-12 and SRQ-20) in Brazil, using relative operating characteristic (ROC) analysis. Psychol Med 1985;15:651-9.

40 Whitlatch CJ, Zarit SH, von Eye A. Efficacy of interventions with caregivers: a reanalysis. Gerontologist 1991;31:9-14.

41 Zarit SH, Reever KE, Bach-Peterson J. Relatives of the impaired elderly: correlates of feelings of burden. Gerontologist 1980;20:649-55.

42 Zarit SH, Todd PA, Zarit JM. Subjective burden of husbands and wives as caregivers: a longitudinal study. Gerontologist 1986;26:260-6.

43 Dewey ME, Copeland JR. Diagnosis of dementia from the history and aetiology schedule. Int J Geriatr Psychiatry 2001;16:912-7.

44 Kaufer DI, Cummings JL, Ketchel P, et al. Validation of the NPI-Q, a brief clinical form of the neuropsychiatric inventory. J Neuropsychiatry Clin Neurosci 2000;12:233-9. 